\title{
Grammatiker, Humanisten und Moralisten
}

\section{Strosetzki, Christoph}

First published in:

Strosetzki, Christoph (Hrsg.): Geschichte der spanischen Literatur. Tübingen : Niemeyer, 1991, S. 214-225

ISBN: 3-484-50307-6

(C) 1991 Max Niemeyer Verlag GmbH \& Co. KG, Tübingen 


\section{Grammatiker, Humanisten und Moralisten}

Zahlreich sind die Beziehungen, die zwischen der religiösen und der humanistischen Literatur bestanden. So teilte jeder Humanist mit dem Theologen das Bemühen um das Verständnis von besonders gehaltvollen Texten: Während der Theologe die Bibel interpretierte, setzte sich der Humanist mit der antiken Literatur auseinander. Beide waren bemüht, einen Kanon der wertvollen Bücher aufzustellen, diese zu tradieren und zu'kommentieren. Dieser positiven Bewertung der als lesenswert erachteten Bücher stand die Verurteilung der wertlosen, die der Humanist durch Mißachtung und der Theologe durch die Zensur der Inquisition strafte, gegenüber. Die Parallelen der Aufgaben von Grammatikern und Theologen bei der Kanonbildung zeigten sich deutlich bei Fray Luis de Alarcón, der in seinem Camino del cielo (1547) gemäß augustinischer Tradition zwei Typen von Phänomenen in der Welt unterschied. Die einen, die er, die beliebte Metapher der Welt als Buch aufgreifend, Bücher Gottes nannte, ließen sich als Werke Gottes erkennen, die anderen, die er als Bücher des Teufels bezeichnete, als Werke und Instrumente des Teufels. Zu beiden Typen von Phänomenen gehörten natürlich auch, unter anderem, die entsprechenden gedruckten Bücher. Vor diesem Hintergrund wird verständlich, $\mathrm{da} ß$ sich die Inquisition der Grammatiker als Gutachter bedienen konnte, zu deren traditionellen Aufgaben Textkritik und Textbewertung gehörten.

Diese Allianz von Theologen und Grammatikern hatte zur Folge, daß man im Rückgriff auf die Horaz'sche Forderung, in der Literatur das Belehrende mit dem Unterhaltenden zu verbinden, die religiöse Literatur der weltlichen vorzog. In der weltlichen Literatur war es wiederum die belehrende, d.h. vor allem die humanistische, die der bloß unterhaltsamen Literatur, z.B. den Liebes- oder Ritterromanen, von Seiten der Theoretiker vorgezogen wurde. Dies hatte dann zur Folge, daß die Autoren der unterhaltsamen Literatur eine belehrende und nützliche Komponente ihrer Werke zumindest in den Vorworten in den Vordergrund stellten. Wenn man schon kein religiöser Autor war, wollte man doch zumindest als poeta enuditus gelten.

Die Theologen hatten seit dem Mittelalter eine Bibelhermeneutik zur Verfügung, deren Grundlagen nunmehr den Humanisten bei ihrer Kunst der Textauslegung dienten. Diese Hermeneutik als Lehre von der Sinnentschlüsselung konnte auf Elemente der Rhetorik zurückgreifen, mit deren Hilfe Texte verfaßt und verschlüsselt wurden. Insbesondere in der Predigt verbanden sich Bibelauslegung und Rhetorik. Universales Wissen von den in einer Rede möglicherweise vorkommenden Gegenständen wurde seit der Antike vom Redner gefordert. Universales Wissen wird daher auch ein Postulat für die Textauslegung des $\mathrm{Hu}-$ manisten.

Theologe und Humanist erörtern moralphilosophische Fragen und vermitteln ethische Verhaltensnormen. Der Stoizismus stellt dabei unter dem Einfluß 
Senecas $^{1}$ die Grundhaltung für die christlichen und die humanistischen Lehren zur Verfügung. Die Humanisten hoffen, insbesondere in der Antike Auskünfte über Fragen zum Menschen zu finden. Ihre Beschäftigung mit der antiken Literatur verhilft dieser dabei zu einer Renaissance.

Die Auseinandersetzung mit Texten war seit Quintilian ein Anliegen der Grammatiker, die nicht nur zum richtigen Sprachgebrauch erziehen sollten, sondern mit der poetarum enarratio die vorliegende Literatur auch zu deuten hatten. Die in ethischer und sprachlicher Hinsicht vorbildlichen Texte wurden in einem Kanon zusammengestellt, der den Schülern und Lesern zur Nachahmung dienen sollte. Daher bezog die Grammatik als Wissenschaft ihren Rang nicht zuletzt aus dem Glanz ihres Gegenstandes, einer blühenden Literatur oder einer bedeutenden Sprache. Auch aus diesem Grund war der Grammatiker am Zustandekommen eines literarischen Kanons auf seinem Gebiet interessiert. Dieses Interesse teilte er mit dem Humanisten, der nicht nur um die Texte in den alten Sprachen, sondern auch um die seiner eigenen Sprache verfaßten bemüht war.

Als Konkurrent wurde der in der lateinischen und griechischen Sprache versierte Grammatiker von den Theologen angesehen, deren sprachliche Kompetenz bei der Lektüre der Originaltexte der Bibel er übertraf. Seinerseits war es der traditionelle Grammatiker, der im humanistischen Schriftsteller einen Konkurrenten sah.

Seit dem Mittelalter war man es gewohnt, sich in der wissenschaftlichen Literatur der lateinischen Sprache zu bedienen. Daran wurde auch in der Renaissance noch festgehalten, wenngleich gerade die Humanisten Texte, die für ein breiteres Publikum bestimmt waren, in der Sprache ihres eigenen Landes verfaßten. Bei dem bereits im Kontext des 15. Jahrhunderts erwähnten, bekanntesten Grammatiker und Humanisten Antonio de Nebrija (1442-1522) ${ }^{2}$ ging die Beschäftigung mit dem Lateinischen dem Interesse am Spanischen voraus. Er hatte mit 19 Jahren Spanien verlassen, um seine Ausbildung in Italien abzurunden, wo er seine Kenntnisse der antiken Autoren vertiefte. Nach Spanien zurückgekehrt, lehrte er an der Universität von Salamanca als grammaticus, "poesía, oratoria (de la que formarían parte gramática, retórica, escritos científicos y quizá filosofía moral), historia, jurisprudencia y literatura cristiana" ${ }^{13}$, Disziplinen, mit denen er nicht als Fachmann, sondern als Grammatiker konfrontiert werde. Mit diesem Anspruch verteidigte er sich auch 1499 gegen die Anschuldigungen des Hauptinquisitors Diego de Deza, der ihm vorwarf, in seiner Quinquagena kontroverse Stellen der Bibel grammatisch kommentiert zu haben. ${ }^{4}$ Später veröffentlichte er weitere Bibelkommentare $(1515,1516)$ sowie Arbeiten zur Orthographie (1517) und zur Rhetorik (1515). Er betätigte sich als Herausgeber und

\footnotetext{
1 Vgl. Blüher, K.A., Seneca in Spanien, München 1969.

${ }^{2}$ Vgl. F. Rico, Nebrija frente a los bárbaros, Salamanca 1978; V. García de la Concha (Hg.), Nebrija y la introducción del Renacimiento en España, Salamanca 1983.

3 Zit. nach Schmidt-Braselmann, Petra, Humanistische Grammatik und Volkssprache. Zur 'Gramática de la lengua castellana' von Antonio de Nebrija, Düsseldorf 1989, (Habil., im Druck) 
Kommentator antiker und spätantiker Werke und schrieb, in Nachbardisziplinen eingreifend, einen Traktat über die Kindererziehung (1509), ein Medikamentenlexikon (1518), die Aenigmata Juris Civilis (1506) und ein historisches Werk über die spanischen Antigiledades (1499).

Nebrijas, nach ihrem ersten Erscheinen in vielen Nach- und Raubdrucken verbreitete, Introductiones latinae (1480) beschäftigten sich mit der lateinischen Sprache, während er 1492 mit der Gramática de la lengua castellana die erste vollständige und systematische Beschreibung des Kastilischen publizierte. Mit ihr setzten sich im 16. Jahrhundert zahlreiche andere Grammatiken und Erörterungen auseinander. Juan de Valdés bezweifelt in seinem assoziativen und geistreichen Diálogo de la lengua (1535) ganz generell die Möglichkeit eines grammatischen Regelsystems für das Kastilische, da es aus mehreren Volkssprachen zusammengesetzt sei. Wie Nebrija nimmt Juan Luis Vives zwischen 1519 und 1538 eine sprachliche Entwicklung des Spanischen an und sieht dessen Vorläufer im Lateinischen und im Griechischen. Anders als Nebrija jedoch interessiert ihn nicht der Hintergrund einer translatio imperii, sondern die Geschichtsgebundenheit der Sprache und ihre Entwicklung. Cristóbal de Villalón (1505-1558) macht sich im Vorwort seiner an Nebrija orientierten Gramática castellana (1558) dessen Engagement für die Volkssprache zu eigen, kritisiert aber Nebrijas zu enge terminologische Orientierung am Lateinischen.

$\mathrm{Da}$ nicht allein eine Grammatik für das Prestige erforderlich ist, sondern auch die Rhetorik zur Verfeinerung der Sprachpraxis sowie eine Reihe von Musterautoren, deren Werke sprachliche Maßstäbe setzen, betont Ambrosio de Morales in seinem Discurso de la lengua castellana (1585). Da es in der spanischen Volkssprache lange Zeit keine Texte gegeben habe, die man hätte nachahmen können, und schlechte Liebesgeschichten und Unterhaltungsromane dem Ansehen der Sprache geschadet hätten, hätten gute Schriftsteller die Volkssprache gemieden. Diese Situation habe sich seit einiger Zeit geändert. Als Beispiele für die neuen Musterautoren nennt er Pedro Mexía, Hernando del Pulgar, Alejo Venegas, Francisco Cervantes de Salazar, Luis de Granada und Juan Luis Vives. Es zeigt sich also, daß es in erster Linie Humanisten sind, die mit ihrer Literatur das Kastilische zu neuem Rang erheben. Ihre Werke erscheinen Morales als Wegbereiter für eine gelehrte volkssprachliche Literatur und damit in der Rolle, die Ciceros Schriften für die lateinische Sprache hatten. Auf einige von ihnen soll im folgenden näher eingegangen werden.

Da die Rhetorik als Fortsetzung der Grammatik in der Sprachpraxis gedacht war, ist es naheliegend, daß Grammatiker und Humanisten auch das praktische Verhalten des Einzelnen in der Gesellschaft erörterten. Sie schrieben Verhaltenslehren mit sprachlichen und taktischen Ratschlägen insbesondere für den Höfling, aber auch für zahlreiche andere Berufsgruppen. Anliegen der Humanisten war es bekanntlich, den Menschen in den Mittelpunkt ihrer Betrachtungen zu stellen. In erster Linie diesem Zweck waren ihre schriftstellerischen 
Bemühungen gewidmet. Der Humanist Juan Lorenzo Palmireno (1524-1579) ${ }^{5}$ sieht sich als Grammatiker und bezeichnet seine Tätigkeit als "profesión de enseñar letras humanas", wenn er in El estudioso cortesano (1573) die Agibilia lehrt, zu denen er den sprachlichen Umgang ebenso zählt, wie die Fähigkeit, sich ins rechte Licht $\mathrm{zu}$ setzen. Aus dieser von den Humanisten eingeführten Wissensdisziplin ist die Reflexion von Moralisten wie Baltasar Gracián über das angemessene Verhalten in der Gesellschaft entstanden, das sich nicht nur an moralischen Werten orientiert, sondern auch im Bereich des sprachlichen Verhaltens gemäß dem rhetorischen Postulat des aptum optimale Effizienz anstrebt. Im Zusammenhang mit den Agibilia entstehen zahlreiche Schriften, in denen Fragen der Staatsform, der Erziehung, des Verhaltens von Fürsten, Grammatikern, Mönchen, Frauen oder Höflingen angeschnitten werden; zudem werden darin der Sinn des Adels, die Bedeutung der Liebe bzw. die Gegensätze zwischen kontemplativem und aktivem Leben, zwischen Hof und Land, zwischen Waffenhandwerk und literarischer Bildung sowie zwischen Müßiggang und Arbeit erörtert. $^{6}$

Es läßt sich also eine Entwicklung in der Tätigkeit des Grammatikers beobachten, die von der Kommentierung und kritischen Edition von Texten über die Bücherbewertung und Zusammenstellung von ausgewählten Zitaten hinführt zur Benutzung der so gewonnenen Materialsammlungen und Hintergrundinformationen zu eigenen Texten. Dabei handelt es sich um Dialoge und essayistische Abhandlungen zu unterschiedlichsten Themen. Zu ihnen gehören z. B. ebenso Pedro Mexías (1497-1551) Silva de varia lección (1540) wie seine Coloquios (1547). Mexía wurde 1548 Hofchronist. Er stand in brieflichem Kontakt mit Erasmus, von dem er, wie die meisten spanischen Humanisten des 16. Jahrhunderts, beeinflußt war. Seine in der Struktur an Aulus Gellius' Noctes Atticae orientierte, aus Übernahmen von antiken Autoren zusammengesetzte Silva erwies sich als Bestseller, der in 150 Jahren $33 \mathrm{Mal}$ neu aufgelegt wurde. Auch seine in der beliebten Dialogform verfaßten Coloquios wurden ein Bucherfolg, wie zahlreiche Neuauflagen und Übersetzungen belegen. Während in der Silva Themen, wie die Geschichte des Buches, der Schrift und des Papiers, erörtert werden, kündigen die Kapitelüberschriften der Coloquios Gespräche über Ärzte, Einladungen, die Sonne, die Natur und die Erde sowie ein Gespräch mit einem, der immer recht haben will, an. Die Dialogform erlaubt es den unterschiedlichen Gesprächspartnern, zahlreiche gelehrte Erkenntnisse einzufügen. ${ }^{7}$

Da sich die Gesprächspartner meist hinsichtlich Herkunft und Vorwissen unterscheiden, können Sachverhalte aus wechselnden Perspektiven betrachtet werden. Die Fiktion des Gesprächs hat zudem den Vorzug einer aufgelockerten

\footnotetext{
5 Vgl. Gallego Barnés, Andrés, Juan Lorenzo Palmireno (1524-1579). Un humanista aragonés en el Studi General de Valencia, Zaragoza 1982.

${ }^{6}$ Vgl. Strosetzki, Christoph, Literatur als Beruf. Zum Selbstverständnis gelehrter und schriftstellerischer Existenz im spanischen Siglo de Oro, Düsseldorf 1987, S. 277ff.

7 Vgl. Castro Díaz, Antonio, Los 'Coloquios' de Pedro Mexía. Un género, una obra y un humanista sevillano del siglo $X V I$, Sevilla 1977, S. $105 \mathrm{ff}$.
} 
und zwanglos assoziativen Präsentation der Gedanken, die gerade dem breiteren Laienpublikum entgegenkam, das die Humanisten als Leser gewinnen wollten. Die assoziative und unsystematische Anordnung des Wissens, wie sie im Dialog modellhaft vorliegt, prägte auch andere Darbietungsformen, wie z.B. Mexías Silva, den Escolástico von Villalón, die Miscelánea von Luis Zapata (1526-1595), die Filosofia vulgar von Juan de Mal Lara (1524-1571), aber auch die Sammlung bemerkenswerter Aussprüche von Melchor de Santa Cruz (1520-1580) Floresta española de apothegmas o sentencias, sabia y graciosamente dichas... (1574). ${ }^{8}$ Letztere ist den Adagia $(1500 ; 1515)$ des Erasmus von Rotterdam vergleichbar. Derartige Sammlungen von Sprichwörtern oder Sentenzen haben einen Ursprung in der Gewohnheit der Grammatikschüler, bei der Lektüre die besten und bedeutendsten Sätze der gelesenen Autoren in eigenen Heften niederzuschreiben. Diese wurden als loci communes-Hefte bezeichnet, da ihre immer wieder notierten Sätze zu Gemeinplätzen wurden. Die Apophthegmensammlungen sind also eine literarische Gattung, die sich aus einer verbreiteten Schulpraxis entwickelte.

Die Dialogform ist es, die es dem Humanisten Hernán Pérez de Oliva (1494?-1531?) $)^{9}$ erlaubt, im Diálogo de la dignidad del hombre (1546) Argumente für und wider die Größe des Menschen auszutauschen. Das Gespräch beginnt mit der Darstellung der miseria hominis, die durch die Mühsal der Arbeit belegt wird, und schließt in der Fortsetzung des Herausgebers des Diálogo, Francisco Cervantes de Salazar (1518-1575), mit der für die Renaissance charakteristischen positiven Bewertung des Menschen, der sich durch die Arbeit selbst verwirkliche und zu Ruhm gelange: "De ninguna cosa los hombres ganaron gloria, que no fuese trabajosa."

Dialogform und Bewertungen aus unterschiedlichen Perspektiven charakterisieren auch die beiden Teile des von den Götterdialogen Lukians beeinflußten Diálogo de Mercurio y Carón (1529), in dem zunächst der menschliche Egoismus entlarvt, dann aber der Wert gewissenhafter Pflichterfüllung vorgestellt wird. Sein Autor, Alfonso de Valdés (1490?-1532), war mit Erasmus und Melanchthon befreundet und folgte Karl V. als Sekretär. In seinem Diálogo de las cosas ocurridas en Roma (1529) läßt er die Plünderung der heiligen Stadt Rom durch die kaiserlichen Truppen im Jahr 1527 rechtfertigen, indem er der päpstlichen Kirche durch den Gesprächspartner Lactancio Ämterkäuflichkeit, Habsucht, Ablaßwesen und äußerlichen Pomp anlastet. Sein Bruder, der Humanist Juan de Valdés (1491-1541) wendet sich gleichfalls gegen die verbreiteten religiösen Sitten, die er als Äußerlichkeiten empfindet und denen er ein verinnerlichtes Christentum gegenüberstellt. Unter dem Einfluß Erasmus' von Rotterdam entwirft er seine Vorstellungen dazu im Diálogo de doctrina cristiana (1529) und

8 Vgl. Prieto, Antonio, La prosa española del siglo XVI, Bd. 1, Madrid 1986, S. 48ff.

9 Vgl. Atkinson, W., "Hernán Pérez de Oliva: a Biographical and Critical Study", in: Revue hispanique 71, 1927, S. 309-484.

${ }^{10}$ Cervantes de Salazar, F., Obras que Francisco Cervantes de Salazar ha hecho, glossado y traducido, Madrid 1772, S. 108. 
in Alfabeto cristiano (1546). Die in Zusammenarbeit mit seinem Bruder entstandenen Ciento diez consideraciones divinas (1539) lehren die Suche des Seelenheils durch innere Erleuchtung. Derartige Positionen mußten Juan de Valdés die Verfolgung durch die Inquisition fürchten lassen, zumal da die offizielle Förderung der Lehren des Erasmus in Spanien durch Jiménez de Cisneros endete und die Werke von Erasmus auf den Index gesetzt worden waren. Als Juan de Valdés' Diálogo de doctrina cristiana der Häresie beschuldigt wurde, entzog er sich möglichen Verfolgungen durch seinen Weggang nach Italien. Neben diesen religiös geprägten Werken ist besonders sein bereits erwähnter Diálogo de la lengua bekannt geworden, in dem J. de Valdés getreu der Tradition des Grammatikers nicht nur den Wert der Volkssprache lobt und ihren Gebrauch in einem einfachen und direkten Stil empfiehlt, sondern auch die volkssprachliche Literatur beurteilt und die wertvollen Werke den minderwertigen gegenüberstellt.

So weit war die Volkssprache im Bereich der Wissenschaft schon anerkannt, daß sich ihrer auch der Mediziner Juan Huarte de San Juan (1529?-1588) bediente. In seinem Examen de ingenios para las ciencias (1575) analysiert er ausgehend von der individuell unterschiedlichen Zusammensetzung der Körpersäfte die verschiedenen psychologisch unterscheidbaren Begabungen für unterschiedliche Bereiche der Wissenschaften. Die Erkenntnis des eigenen Charakters wird vor diesem Hintergrund zur Voraussetzung für die richtige Berufswahl. Da diese allgemeinverständliche psychologische Studie einem breiten Leserkreis Selbsterkenntnis und direkten persönlichen Nutzen versprach, wurde sie zu einem Bucherfolg mit zahlreichen Neuauflagen und Übersetzungen.

Anders als Huarte bedient sich der Humanist Juan Luis Vives (1492-1540) ${ }^{11}$ vorzugsweise des Lateinischen. Dies ist jedoch nicht auf seine Geringschätzung des Spanischen, sondern auf seine internationale Orientierung zurückzuführen. Als Christ aus jüdischem Elternhaus mußte er im Ausland Schutz vor der spanischen Inquisition suchen. Er studierte in Paris, war Hauslehrer am englischen Königshof und lehrte in Löwen, Oxford und Brügge. In De causis comuptarum artium setzt er sich mit der aristotelischen Lehre auseinander, die er in der scholastischen Hochburg Paris kennengelernt hatte, und vertritt eine empiristische Theorie. In De subventione pauperum (1526) tritt er für die Armenfürsorge ein, während sein De dissidiis Europae et bello turcico (1526) umfassende außenpolitische Kenntnisse belegt. Davon, daß er als Humanist auch pädagogisch tätig war, zeugt sein der Mädchenerziehung gewidmetes Werk $D e$ institutione feminae christianae (span. Übersetzung 1528). Hier wie in anderen Schriften sieht er in der fiktionalen Literatur nur dann einen Wert, wenn diese Wissen vermittelt oder moralischen Nutzen bietet. Der Gelehrte mit seiner abgeklärten Weisheit verkörpert für ihn die Idealfigur, die er in Introductio ad sa-

\footnotetext{
${ }^{11}$ Vgl. Buck, August (Hg.), Juan Luis Vives, Arbeitsgespräch in der Herzog-August-Bibliothek in Wolfenbiuttel, Hamburg 1982.
} 
pientiam (1524) entwirft. Dieser Text ist 1554 von F. Cervantes de Salazar ins Spanische übersetzt sowie mit Anmerkungen erweitert und kommentiert worden.

Die Kanonisierung einer Literatur mit nachahmenswerten Idealfiguren und Verhaltensnormen war schon das Anliegen der Grammatiker gewesen. Nunmehr sind es die Humanisten, die sich verstärkt dieser Aufgabe widmen. Ein Beispiel dafür ist Antonio de Guevara (1480?-1545) ${ }^{12}$. Nach einer Zeit als Page wurde er am königlichen Hof Franziskaner, Prediger, Chronist und schließlich Bischof. Seine Nähe zum Hof erklärt seine Distanz zu einer ihm pedantisch erscheinenden Exaktheit der universitären Textkritik und Zitierweise. Demgegenüber geht Guevara als mondäner Schriftsteller bewußt "dilettantisch" und freizügig bei der Bearbeitung der ihm zur Verfügung stehenden Textmaterialien vor. Ungenaue Zitierweisen, falsche Autorenzuschreibungen sowie Erfindungen von Zitaten und Autoren trugen ihm schließlich die Vorwürfe des Grammatikers Pedro de Rúha ein, die in den Cartas de Rhúa lector en Soria sobre las obras del Rev. señor Obispo de Mondoñedo (1549) nachzulesen sind.

Dem Hof nahestehend, schrieb Guevara zunächst einen vielgelesenen und vielfach übersetzten Fürstenspiegel in Briefform, in dessen Mittelpunkt er als Vorbild den weisen Stoiker Marc Aurel stellte: Libro áureo de Marco Aurelio (1528), der zunächst unautorisiert und anonym erschien, bevor der Autor ihn ergänzte und im darauf folgenden Jahr unter dem Titel Libro llamado relox de príncipes erscheinen ließ. Auf die bewährte Briefform kam Guevara später noch einmal in den an Cicero orientierten Essays über unterschiedlichste Themen Epistolas familiares (1. Teil: 1539, 2. Teil: 1541) zurück, während er in seiner Década de Césares (1539) weitere Biographien römischer Herrscher zusammenstellte.

Von besonderem Interesse ist sein Libro llamado auiso de privados y doctrina de cortesanos (1539). Hier beschreibt er nämlich Normen für die effiziente Dialogführung des auf Karriere bedachten Höflings. Er entspricht damit der von den Grammatikern erhobenen Forderung nach Vervollkommnung der sprachlichen Praxis durch die Rhetorik ebenso wie der Aufstellung von Verhaltensnormen, die er allerdings weniger an der christlichen Moral orientiert als am rhetorischen Postulat des aptum. Analysiert wird, welchen Sinn ein Gespräch haben soll, wie der Sprecher und seine Gesprächspartner einzuschätzen sind, welche Redelänge und -häufigkeit empfohlen wird und schließlich, wie Lüge, Schmeichelei, üble Nachrede und Spott einzuschätzen sind.

Mit der Abkehr von einer ausschließlich religiös orientierten Moralisierung und mit dem Interesse an Normen für effizientes Verhalten leitet er als humanistisch geprägter Schriftsteller eine Entwicklung ein, die beim Moralisten B. Gracián einen Höhepunkt erreichen wird. Während bei Vives Ratschläge zur Gesprächsführung unter Bezug auf die Kirchenväter und die Antike religiös und ethisch fundiert sind, deutet sich die bei Guevara beobachtete Säkularisierung 
auch unter italienischem Einfluß in Juan Boscáns (1487?-1542) ${ }^{13}$ Übersetzung von Castigliones Cortegiano und in Lucas Gracián Dantiscos Galateo español (1590) an, der den italienischen Galateo von Della Casa auf spanische Verhältnisse übertragen wollte. ${ }^{14}$

$\mathrm{Da}$ das Leben des Höflings nur schwer mit den Interessen des humanistischen Schriftstellers zu verbinden ist, wird in Menosprecio de corte y alabanza de aldea (1539) deutlich. Hier greift Guevara auf den antiken Topos des Beatus ille zurück, der denjenigen glücklich preist, der sich fern von den Verpflichtungen des Hofes oder politischer Geschäfte ungestört und ungezwungen, in guter Luft auf dem Land seinen literarischen Studien widmen kann. Es kann aber auch sein, daß Guevara mit dieser Schrift nicht beabsichtigt, einen humanistischen Lebensstil zu verteidigen, sondern daß er der damals bedrohliche Ausmaße annehmenden Landflucht Einhalt gebieten möchte. Die Tatsache, daß sein Spätwerk durch religiöse Schriften charakterisiert ist, verbindet ihn mit den ein Jahrhundert später lebenden Moralisten Francisco Quevedo und B. Gracián, die im folgenden vorgestellt werden sollen.

Die Moralisten beschäftigten sich, ähnlich wie die Humanisten, in erster Linie mit den menschlichen Verhaltensformen (mores). Wenn sie diese erörterten oder mit Beispielen veranschaulichten, erhoben sie jedoch nicht den moralischen Zeigefinger, um zur Einhaltung ethischer und religiöser Normen aufzufordern, sondern beschränkten sich auf die Thematisierung faktisch bestehender Sitten. Diese führten sie, wie Quevedo in seinen "Sueños", satirisch und kritisch vor, oder sie leiteten - wie Gracián - ganz affirmativ zur Optimierung des Verhaltens im gegebenen Kontext an.

Der mit seinem Schelmenroman El Buscón bereits vorgestellte, ${ }^{15}$ humanistisch gebildete Francisco Gómez de Quevedo y Villegas (1580-1645) wurde nach einem Theologiestudium hoher Beamter, Diplomat und Sekretär Philipps IV., bevor er infolge einer Intrige in Ungnade fiel. Von seinen zahlreichen religiösen, lyrischen, politischen, satirischen und literaturkritischen Werken können nur wenige erwähnt werden. Mit Góngora war er durch einen literarischen Streit verbunden, den er von 1603 bis zu dessen Todesjahr 1627 führte. Scharf ist seine Polemik z.B. in der Prosasatire Aguja de navegar cultos (1613) gegen Góngoras mit reichem rhetorischen Schmuck ausgestattete culteranistische Lyrik, ${ }^{16}$ die er als Kauderwelsch bezeichnet. Für die Abfassung eines solchen Gedichts an einem einzigen Tag weiß er, ein geeignetes Rezept anzubieten.

Quevedo leistete einen bedeutenden Beitrag zur Tradition der Traktate über das rechte Verhalten des Fürsten. Diese auch Fürstenspiegel genannten Schriften "de regimine principum" sind in Spanien während des Siglo de Oro zu großer

\footnotetext{
${ }^{13}$ Vgl. auch hier: G. Güntert, Siglo de Oro: Lyrik, Teil I., Kap. 2.1., S. 124f.

${ }^{14} \mathrm{Vgl}$. Strosetzki, Chr., "Sprache als Handlung in der spanischen Renaissance", in: Wolfenbïtteler Renaissance-Mitteilungen 5/1, 1981, S. 43-49.

${ }^{15}$ Vgl. hier: Chr. Strosetzki, Der Roman im Siglo de Oro, Kap. 8., S. $111 f$.

${ }^{16}$ Vgl. auch hier: G. Güntert, Siglo de Oro: Lyrik, Teil II., Kap. 3., S. $149 f$ f.
} 
Blüte gelangt. Sie greifen auf die Antike und auf Thomas von Aquin zurück. Nicht selten dienen sie der Auseinandersetzung mit Niccold Machiavellis Il principe (1532), dessen verabsolutierte Staatsraison sie ablehnen. Diese Haltung charakterisiert auch Quevedos Polltica de Dios, gobierno de Cristo, tirania de Satanás $(1626,1655)$. Er geht darin von Christus aus, der als wahrer König nicht nur andere, sondern auch sich selbst beherrsche. Wie ihn andere Herrscher nachahmen sollen, legt er mit Vergleichen aus der Bibel dar. Er zeigt, wie sie Gerechtigkeit und Frieden sichern, aber auch, wie sie sich kleiden oder Schmeichlern gegenüber verhalten sollen.

Während er mit seinem Fürstenspiegel złr Nachahmung positiv bewerteter Normen auffordert, erscheinen seine Sueños $(1627)^{17}$ als Gesellschaftssatire, in der die verbreitete Befolgung falscher Normen zum Teil vor dem Hintergrund der "letzten Dinge" angeprangert wird. Durch den ernsthaften Rahmen wird das Thema der Weltverachtung eingeführt und bloß vordergründige Komik durch eine didaktische Komponente relativiert. Es handelt sich um Traumvisionen, deren satirische Gestaltung Lukians Göttergesprächen nachempfunden ist. Der Einleitung dienen erzählende Elemente, in denen der Dichter, der an den folgenden Gesprächen selbst kaum beteiligt wird, Protagonist ist. Die durch Karikatur, Hyperbolik, Wortspiele und Pointen erzielte Satire gilt einzelnen Personen ebenso wie der Politik, der Literatur und der Sprache. Die einzelnen Sueños sind von 1606 bis 1622 entstanden: El juicio final, El alguacil endemoniado, El sueño del infierno, El mundo por de dentro und El sueño de la muerte. Die Titel wurden verschiedentlich mit Rücksicht auf die Zensur abgewandelt. Diesen Sueños hatte man aus anderen Werken des Autors noch bis zu drei weitere hinzufügen wollen. In El juicio final treten Repräsentanten unterschiedlichster gesellschaftlicher Gruppen auf: Nachdem ein Engel die Toten geweckt hat, treten Geizhälse, Ärzte, Schneider, Kleriker, Apotheker und leichte Mädchen auf, die vor Jupiters Thron vorgelassen werden und für ihr vergangenes Verhalten Erklärungen abgeben. So tritt neben die Standessatire die der Charaktere. In El alguacil endemoniado beklagt sich ein Geist, daß er in der Haut eines Gerichtsvollziehers Unterschlupf finden muß, während in El sueño del infierno die unterschiedlichen Bestrafungen der verschiedenen Berufsgruppen in der Hölle geschildert werden. In El mundo por de dentro schließlich ist es die allegorische Figur der "Desilusión", die die Scheinhaftigkeit des Reichtums und der Schönheit in einer Welt entlarvt, in der jene Verstellung herrscht, die den Blick auf die Wahrheit versperrt. Einen vergleichbaren Prozeß der Desillusionierung nahm wenige Jahre später Gracián mit seinem Criticón (1651-1657) vor. ${ }^{18}$

Der einer Lehrtätigkeit nachgehende Jesuit B. Gracián (1601-1658), dessen einziges theologisches Werk El comulgatorio (1655) war, mußte seine gleichermaßen an der Rhetorik orientierten und für das höfische Leben konzipierten

${ }^{17} \mathrm{Vgl}$. Nolting-Hauff, Ilse, Vision, Satire und Pointe in Quevedos "Sueños", München 1968

${ }^{18}$ Der Criticón wurde im Zusammenhang mit dem Roman des Siglo de Oro bereits vorgestellt. 
Schriften mit dem Pseudonym Lorenzo Gracián veröffentlichen, um möglichen Anfeindungen seiner Ordensbrüder zu entgehen. Für die von ihm modellhaft in den meisten Werken entworfenen Idealfiguren stellt er Maximen, Verhaltensweisen und Denkmuster vor, mit denen sie in dem ihnen zur Verfügung stehenden Rahmen optimale Effizienz erreichen:

Hombre en su punto. No se nace hecho; vase de cada día perficionando en la persona, en el empleo, hasta llegar al punto del consumado ser, al complemento de prendas, de eminencias: conocerse ha en lo realzado del gusto, purificado del ingenio, en lo maduro del juicio, en lo defecado de la voluntad. Algunos nunca llegan a ser cabales, fáltales siempre un algo; tardan otros en hacerse. El varón consumado, sabio en dichos, cuerdo en hechos, es admitido y aun deseado del singular comercio de los discretos.

Ein möglicher Konflikt zwischen zweckorientierter Taktik und christlicher Moralität ist in diesem Ansatz immer latent vorhanden. Gracián umgeht ihn dadurch, daß er die religiöse von der weltlichen, in seinen Werken im Vordergrund stehenden Sphäre getrennt wissen will. "Hanse de procurar los medios humanos como si no hubiese divinos, y los divinos como si no hubiese humanos: regla de gran maestro, no hay que añadir comento. ${ }^{20}$

In seiner schematisch nach einem Tugendraster aufgebauten und idealisierten Fürstenbiographie El polltico don Fernando el Cathólico (1640) stellt er das kluge Verhalten des Königs Ferdinand allen ihm folgenden Herrschern als nachzuahmendes Vorbild vor. Dabei handelt es sich nicht nur um eine der rhetorischen Gattung der Lobrede entsprechende Huldigung Ferdinands, sondern zugleich um eine Zusammmenstellung der wichtigsten Voraussetzungen und Qualitäten eines jeden guten Herrschers, die im einzelnen exemplifiziert werden.

Um Idealfiguren geht es auch in anderen Schriften Graciáns: El héroe (1647) stellt ausgehend von Seneca, Äsop, Homer, Aristoteles und Tacitus, die als Quellen genannt, aber nicht mit Stellenangabe und zum Teil bewußt ingeniös verändert zitiert werden, die Fähigkeiten und Eigenschaften der Idealfigur schlechthin vor. Dazu gehören z.B.: "medir el lugar con su artificio", "cifrar la voluntad", "entendimiento", "corazón de rey", "gusto relevante", "eminencia en lo mejor" und "excelencia de primero". Derartige Qualitäten werden in den einzelnen Abschnitten in knappen und prägnanten Sätzen vorgeführt, die zum Teil rhetorisch in ihrer Kürze so verschlüsselt sind, daß man sie erschließen muß. Diese Art des Stils wurde als konzeptistisch bezeichnet und den langatmigen, mit viel rhetorischem Schmuck ausgestatteten Sätzen Luis de Góngoras gegenübergestellt, die als kulteranistisch gelten. Allerdings wird diese Unterscheidung bei genauerer Analyse unscharf. ${ }^{21}$

Die Knappheit der Sätze Graciáns findet ein Vorbild in der im Barockzeitalter beliebten Emblematik und der Kunst der Devise, bei der einer bildlichen Darstellung, die meist eine Tugend verkörperte, ein knapper Satz zugeordnet

\footnotetext{
${ }^{19}$ Gracián, Baltasar, Obras completas, A. del Hoyo (Hg.), Madrid (Aguilar) 1967, S. 154

${ }^{20}$ Ebda., S. 218. "Gran maestro" bezieht Gracián auf den Ordensgründer Ignatius von Loyola, von dem P. Pedro de Rivadeneyra in dessen Biographie einen ähnlichen Satz überliefert.

${ }^{21}$ Vgl. hier: G. Güntert, Siglo de Oro: Lyrik, Teil II, Kap. 1., S. 147.
} 
wurde. Derartige Devisen, die insbesondere Fürsten zu feierlichen Anlässen als Motti ihrer Herrschaft gern zur Schau trugen, wurden auch in Fürstenspiegeln benutzt. Ihre Spuren lassen sich bei Gracián entdecken. Deutliches Strukturprinzip sind sie im antimachiavellistischen Fürstenspiegel des Diplomaten Diego de Saavedra Fajardo Idea de un príncipe politico-cristiano, representada en cien empresas (1640).

Ihre Bedeutung unterstreicht Gracián in seinem poetologischen Werk Agudeza y arte de ingenio (1642), in dem er sie mit Perlen vergleicht, die trotz ihrer geringen Größe von hohem Wert sind. Sie sind für sein Verständnis des Konzeptismus ebenso paradigmatisch wie das Bemühen darum, das Wesentliche zunächst nicht offenzulegen: "Cuanto más escondida la razón, y que cuesta más, hace más estimado el concepto, despiértase con el reparo la atención, solicítase la curiosidad, luego lo exquisito de la solución desempeña sazonadamente el misterio." ${ }^{\prime 22}$ Gracián selbst unterstreicht die Parallelen zwischen seiner Poetik und den Regeln der Rhetorik. Da es nun auch Regeln der Rhetorik sind, die der Höfling im gesellschaftlichen Umgang und in der Konversation zu berücksichtigen hat, wundert es nicht, daß in Graciáns Schrift über das Verhalten des Höflings vergleichbare rhetorische Regeln anzutreffen sind. So findet man in Graciáns El discreto (1646) jene Agibilia wieder, die schon J.L. Palmireno thematisiert hatte. Der in der "Agudeza" als Rätsel vor einem zu entlarvenden Kern geschickt täuschende Schein einer schriftstellerischen Aussage begegnet auch in der zwischenmenschlichen Interaktion als zu nutzendes oder zu durchschauendes taktisches Mittel.

Hacer y hacer parecer. Las cosas no pasan por lo que son, sino por lo que parecen. Valer y saberlo mostrar es valer dos veces: lo que no se ve es como si no fucse. No tiene su veneración la razón misma donde no tiene cara de tal. Son muchos más los engañados que los ajvertidos; prevalece el engaño y júzganse las cosas por fuera; hay cosas que son muy otras de lo que parecen. La buena exterioridad es la mejor recomendación de la perfección interior. $^{23}$

Mit den Humanisten des vorausgegangenen Jahrhunderts teilt Gracián die Gewohnheit, zentrale Sätze aus unterschiedlichen Werken zu sammeln und kommentiert zu veröffentlichen. Sein von A. Schopenhauer ins Deutsche übersetzter Oráculo manual y arte de prudencia (1647) steht somit in der Tradition der humanistischen Apophthegmatasammlungen. Allerdings entstammen Graciáns Sentenzen, die er in den einzelnen Aphorismen kommentiert oder amplifiziert, im allgemeinen seinen eigenen Werken. Dies belegt erneut die enge Verbindung zwischen den Anliegen der Grammatiker und Humanisten des 16. Jahrhunderts und jenen der Moralisten des 17. Jahrhunderts.

\footnotetext{
${ }^{22}$ Gracián, Baltasar, Obras completas, S. 266.

${ }^{23}$ Ebda., S. 188
} 


\section{Bibliographie:}

Bahner, Werner, Beitrag zum Sprachbewußtsein in der spanischen Literatur des 16. und 17. Jahrhunderts, Berlin 1956

Bataillon, Marcel, Erasmo y el Erasmismo, Barcelona 1977

Bataillon, Marcel, Erasmo y España. Estudios sobre la historia espiritual del siglo XVI, Mexico, Madrid, Buenos Aires 1979

Batllori, Miguel, Gracián y el Barroco, Rom 19.58

Buck, August, Die humanistische Tradition in der Romania, Bad Homburg, Berlin, Zürich 1968

Elias, Norbert, Uber den Proze $\beta$ der Zivilisation. Soziogenetische und psychogenetische Untersuchungen, Frankfurt 1977, 2. Aufl., 2 Bde. (Die Situation in Frankreich zum Vergleich mit Parallelen zu Spanien)

Jansen, Hellmuth, Die Grundbegriffe des Baltasar Gracián, Genf/Paris 1958

Krauss, Werner, Graciáns Lebenslehre, Frankfurt 1947

Kristeller, Paul Oskar, Humanismus und Renaissance, München 1976, 2 Bde.

Maravall, José Antonio, Antiguos y modemos. La idea de progreso en el desarrollo inicial de una sociedad, Madrid 1966

Maravall, José Antonio, Carlos V. y el pensamiento político del Renacimiento, Madrid 1960

Maravall, José Antonio, Estado moderno y mentalidad social. Siglos XV a XVII, 2 Bde., Madrid 1972

Porqueras Mayo, Alberto, La teoría poética en el renacimiento y manierismo españoles, Barcelona 1986

Rothe, Arnold, Quevedo und Seneca. Untersuchungen zu den Frühschriften Quevedos, Genf/Paris 1965

Schröder, Gerhart, Baltasar Graciáns "Criticón", München 1966

Schulte, Hansgerd, El desengaño. Wort und Thema in der spanischen Literatur des Goldenen Zeitalters, München 1969

Sobejano, Gonzalo (hg.), Francisco de Quevedo, Madrid 1978

Stupperich, Robert, Erasmus von Rotterdam und seine Welt, Berlin, New York 1977

Torre, Esteban, Sobre lengua y literatura en el pensamiento científico español de la segunda mitad del siglo $X V I$, Sevilla 1984 